\title{
THE GERBER-SHIU EXPECTED DISCOUNTED PENALTY-REWARD FUNCTION UNDER AN AFFINE JUMP-DIFFUSION MODEL
}

\author{
BY
}

Florin AVRam AND Miguel UsAbel

\begin{abstract}
We provide a unified analytical treatment of first passage problems under an affine state-dependent jump-diffusion model (with drift and volatility depending linearly on the state). Our proposed model, that generalizes several previously studied cases, may be used for example for obtaining probabilities of ruin in the presence of interest rates under the rational investement strategies proposed by Berk \& Green (2004).
\end{abstract}

\section{KEYWORDS}

First passage problems, risk process, stochastic rates of interest, ruin with interest, affine jump-diffusion models, penalty/reward functions at ruin.

\section{INTRODUCTION AND MOTIVATION}

There has been a considerable interest over the last years in obtaining analytical results for ruin problems which take into account a realistic economic environment, including interest rates and investment possibilities.

We consider below a diffusion perturbed risk process

$$
d R_{t}=c d t+\sigma d W_{t}^{(1)}-d X_{t}
$$

where $c$ is the constant premium rate, $X_{t}$ is the total claims compound Poisson process with Lévy measure $\lambda f(x) d x, \lambda$ and $f(x)$ being respectively the intensity and density of the jumps; and $\sigma d W_{t}^{(1)}$ with $W_{t}^{(1)}$, an independent Wiener processes, introduces a source of volatility in the premiums accrual.

We suppose the reserves process $U_{t}$, including investment, is of the form

$$
d U_{t}=\left(\delta+\kappa\left(U_{t}\right) d W_{t}^{(2)}\right) U_{t}+d R_{t}
$$

where the parameter $\delta>0$ and the function $\kappa\left(U_{t}\right)$ model respectively expected instantaneous financial yields (or interest rates) and volatility per monetary unit, 
and $d W_{t}^{(2)}$ is a Brownian motion independent of $X_{t}$ and $d W_{t}^{(1)}$. The case $\delta \leq 0$ (of less applied interest) will not be considered here.

Let us now define $\tau$ as the time to ruin

$$
\tau=\inf \left\{t \geq 0 ; U_{t}<0\right\}
$$

and

$$
v_{t}(u)=\mathbb{E}_{\left\{U_{0}=u\right\}}\left(P\left(U_{t}\right) 1_{\{\tau \geq t\}}+\pi\left(U_{\tau}\right) 1_{\{\tau<t\}}\right)
$$

where $u:=U_{0} \geq 0$ are the initial reserves, denote the expected penalty/reward function. These functions represent respectively:

- The penalty at ruin $\pi\left(U_{\tau}\right)$ with deficit $U_{\tau}, \pi: \mathbb{R}^{-} \rightarrow \mathbb{R}$

- The reward or pay-off on survival after $t$ years: $P\left(U_{t}\right), P: \mathbb{R}^{+} \rightarrow \mathbb{R}$.

In the case $t=\infty$, this becomes the "perpetual/ultimate" penalty/reward:

$$
v(u)=P(\infty) \mathbb{P}_{\left\{U_{0}=u\right\}}\{\tau=\infty\}+\mathbb{E}_{\left\{U_{0}=u\right\}} \pi\left(U_{\tau}\right) 1_{\{\tau<\infty\}}
$$

Some particular cases of interest are the survival probability within $t$ years

$$
\pi\left(U_{\tau}\right)=0, P\left(U_{t}\right)=1_{\left\{U_{t} \geq 0\right\}}
$$

and the ruin probability with deficit larger (absolute value) than $y$

$$
\pi\left(U_{\tau}\right)=1_{\left\{U_{\tau}<-y\right\}}, P\left(U_{t}\right)=0
$$

Previous literature on the topic has usually considered a constant volatility per monetary unit $\kappa\left(U_{t}\right)=\sigma_{r} \geq 0$, and focussed mostly on the deterministic case including the deterministic case $\sigma_{r}=0$. The first passage problem for this class of processes has been treated extensively, for instance, by Segerdahl (1942), Delbaen and Haezendonck (1987), Garrido (1989), Asmussen and Bladt (1996), Paulsen (1993), Embrechts and Schmidli (1994), Peters (1994), Sundt and Teugels (1995), Paulsen and Gjessing (1997a, 1997b), Dickson and Waters (1999), Norberg (1999), Wang and Wu (2001), Cai and Dickson (2002), Kalashnikov \& Norberg (2002), Göing-Jeaschke \& Yor (2003), Novikov (2003), Ma and Sun (2003), Yuen and Wang (2004, 2005), Cai (2004), Cai \& Yang (2005), Gaier \& Grandits (2004), Paulsen, Kasozi \& Steigen (2005) and Gerber \& Yang (2007).

However, despite of the common previous use of the constant volatility per unit rule, $\kappa\left(U_{t}\right)=\sigma_{r}$, this assumption may not be regarded as totally realistic in many rational investments. The fact that the volatility per monetary unit remains constant regardless the size of the fund, $U_{t}$, may be viewed as arguable following financial and insurance markets rationality for several reasons.

The first evidence is a basic lesson from asset allocation strategies. Larger portfolios can afford designing sophisticated hedging strategies through asset 
allocation diversification in stock, fixed-income securities, commodities, derivatives and real estate, among others. Moreover, the increasing transactional costs and insurance supervision authorities regulations may also lead the companies to reduce the actively traded amount, and thus subject to volatility, of the surplus. These facts suggest that for an eventual fixed expected return of $\delta$, the volatility per unit may be a decreasing function of the total size of the fund $U_{t}$.

This intuitive argument is not new, and is supported for example by Berk \& Green (2004). Their model, based on the rationality presented in the former paragraph, successfully explained many salient features of the Mutual Funds flows and performance, previously unexplained in literature. In expression (36) of the cited paper, under the rational model considered, a decreasing volatility per unit of the financial yields with size is proved

$$
\kappa\left(U_{t}\right)=\frac{\sigma_{r}}{\sqrt{U_{t}}}
$$

(see the original work for details). Note that when $\sigma_{r}=0$ is assumed, the constant volatility and Berk \& Green's model for $\kappa\left(U_{t}\right)$ coincide.

Under this model and expanding (1), an affine process is obtained

$$
d U_{t}=\left(c+\delta U_{t}\right) d t+\sqrt{\sigma^{2}+\sigma_{r}^{2} U_{t}} d W_{t}-d X_{t}
$$

Affine processes have been also recently used in many financial application due to their computational tractability and flexibility in capturing many of the empirical features of financial time series; see the comprehensive work by Duffie, Filipovic and Schachermayer (2003) and references therein. The solution of the first-passage problem presented here seems however new.

The affine process $U_{t}$ is Markovian, with infinitesimal generator $\Gamma$

$$
\begin{aligned}
\Gamma v_{t}(u)= & (c+\delta u) \frac{\partial v_{t}(u)}{\partial u}+\left(\frac{\sigma^{2}+\sigma_{r}^{2} u}{2}\right) \frac{\partial^{2} v_{t}(u)}{\partial u^{2}} \\
& +\lambda \int_{0}^{\infty}\left[v_{t}(u-x)-v_{t}(u)\right] f(x) d x
\end{aligned}
$$

The Feymann-Kac formula identifies the expectations of the form (2) with the solutions of the Fokker-Planck equation:

$$
\begin{aligned}
\Gamma v_{t}(u)-\frac{\partial v_{t}(u)}{\partial u} & =0 & & \text { for } u>0 \text { and } t>0 \\
v_{t}(u) & =\pi(u) & & \text { for } u<0 \text { and } t \geq 0 \\
v_{0}(u) & =P(u) & & \text { for } u>0
\end{aligned}
$$


One should notice that $v_{t}(u)$ is not usually continuous at $u=0$ and henceforth $v_{t}(0) \stackrel{d e f}{=} \lim _{u \rightarrow 0^{+}} v_{t}(u)$. In our paper, $v_{t}(u)$ serves only as motivation. Our results concern a smoothed version of $v_{t}(u)$, its Laplace-Carson transform in time, defined as

$$
\Upsilon_{\alpha}(u)=\mathbb{E}_{u}\left(P\left(U_{H_{\alpha}}\right) 1_{\left\{\tau \geq H_{\alpha}\right\}}+\pi\left(U_{\tau}\right) 1_{\left\{\tau<H_{\alpha}\right\}}\right)=\int_{0}^{\infty} \alpha e^{-\alpha t} v_{t}(u) d t
$$

where $H_{\alpha}$ is an exponentially distributed random variable with parameter $\alpha$. Taking a transform in $t$ of (5), and putting $R(u)=\lambda \int_{0}^{\infty} \pi(u-x) f(x) d x$, we find that the Laplace-Carson transform in time of the expected reward satisfies the integro-differential equation

$$
\begin{gathered}
(c+\delta u) \Upsilon_{\alpha}^{\prime}(u)+\left(\frac{\sigma^{2}+\sigma_{r}^{2} u}{2}\right) \Upsilon_{\alpha}^{\prime \prime}(u)+ \\
\lambda \int_{0}^{u} \Upsilon_{\alpha}(u-x) f(x) d x-(\lambda+\alpha) \Upsilon_{\alpha}(u)+R(u)+\alpha P(u)=0 \\
\Upsilon_{\alpha}(0)=\pi\left(0^{-}\right) \quad \text { if } \sigma>0
\end{gathered}
$$

where the latter formula is implied by the presence of Brownian motion, which together with $u=0$ ensure the immediate crossing of 0 - see for example the proof of Theorem 2.1. in Paulsen and Gjessing (1997). The object of our study will be the (7).

Contents: We provide below a unified self-contained treatment of first passage problems under the general affine rational model (4). As mentioned earlier, the result applies to all previously considered cases in literature where the particular case $\sigma_{r}=0$ is assumed.

Section 2 presents the solution existence theorem for the main equation (7) and some useful lemmas. The main theoretical result is contained in section 3, namely a closed formula for the Laplace transform of the exponentially killed expected penalty-reward function in Theorem 2. Finally, some examples and previously obtained cases in literature are revisited in section 4 .

\section{Preliminary Results}

We start with the existence theorem of equation (7). The proof is contained in the Appendix.

Theorem 1. Let $\bar{F} \in C^{2}[0, \infty), P(u)$ and $R(u)$ are bounded for $u \geq 0$ and continuously differentiable on $\left[0, u_{0}\right)$ for $u_{0}$ small enough. Then equation (7) has a solution $\mathrm{Y}_{\alpha} \in C^{2}[0, \infty)$. 
Our first result yields an equation for the Laplace transform of the solutions of (7)

$$
\Upsilon_{\alpha}^{*}(s)=\int_{0}^{\infty} e^{-s u} d_{u} \Upsilon_{\alpha}(u)
$$

Lemma 1. The Laplace transform of a solution of (7) satisfies for $s>0$ the linear $O D E$ :

$$
\begin{aligned}
& -\left(\delta+\frac{\sigma_{r}^{2}}{s} s\right) \Upsilon_{\alpha}^{* \prime}(s)+\left(c+\frac{\sigma^{2} s}{2}-\frac{\sigma_{r}^{2}}{2}-\lambda \bar{F}^{*}(s)-\alpha / s\right) \Upsilon_{\alpha}^{*}(s) \\
& -\Upsilon_{\alpha}(0)\left(\lambda \bar{F}^{*}(s)-\alpha / s\right)-\frac{\sigma^{2}}{2} \Upsilon_{\alpha}^{\prime}(0)+R^{*}(s)+\alpha P^{*}(s)=0
\end{aligned}
$$

where $R^{*}(s)=\int_{0}^{\infty} e^{-s u} R(u) d u$ and $P^{*}(s)=\int_{0}^{\infty} e^{-s u} P(u) d u$.

Proof: Taking the Laplace transform in $u$ of the IDE (7) and using $\int_{0}^{\infty} e^{-s u} \Upsilon_{\alpha}(u) d u=\frac{\Upsilon_{\alpha}^{*}(s)+\Upsilon_{\alpha}(0)}{s}$ we obtain for $s>0$ :

$$
\begin{aligned}
& c \Upsilon_{\alpha}^{*}(s)-\delta \Upsilon_{\alpha}^{* \prime}(s)+\frac{\sigma^{2}}{2}\left(s \Upsilon_{\alpha}^{*}(s)-\Upsilon_{\alpha}^{\prime}(0)\right) \\
& -\frac{\sigma_{r}^{2}}{2}\left(\Upsilon_{\alpha}^{*}(s)+s \Upsilon_{\alpha}^{* \prime}(s)\right)+\lambda s^{-1} f^{*}(s)\left(\Upsilon_{\alpha}^{*}(s)+\Upsilon_{\alpha}(0)\right) \\
& -(\lambda+\alpha)\left(\frac{\Upsilon_{\alpha}^{*}(s)+\Upsilon_{\alpha}(0)}{s}\right)+R^{*}(s)+\alpha P^{*}(s) \\
& =0
\end{aligned}
$$

which yields the result

Let now

$$
B(s)=\delta+\frac{\sigma_{r}^{2}}{2} s, A(s)=c+\frac{\sigma^{2} s}{2}-\frac{\sigma_{r}^{2}}{2}-\frac{\alpha}{s}
$$

so that the ODE (9) becomes:

$$
\begin{aligned}
& -B(s) \Upsilon_{\alpha}^{* \prime}(s)+\left(A(s)-\lambda \bar{F}^{*}(s)\right) \Upsilon_{\alpha}^{*}(s)-\Upsilon_{\alpha}(0)\left(\lambda \bar{F}^{*}(s)+\alpha / s\right) \\
& -\frac{\sigma^{2}}{2} \Upsilon_{\alpha}^{\prime}(0)+R^{*}(s)+\alpha P^{*}(s)=0
\end{aligned}
$$

Recall that the general solution of the equation $\Upsilon_{\alpha}^{*}(s)^{\prime}-C_{1}(s) \Upsilon_{\alpha}^{*}(s)+C_{2}(s)=0$ is $\Upsilon_{\alpha}^{*}(s)=\int_{0}^{\infty} C_{2}(x) e^{-\int_{s}^{x} C_{1}(z) d z}$. Since we must solve: 
$\Upsilon_{\alpha}^{*}(s)^{\prime}-\frac{A(s)-\lambda \bar{F}^{*}(s)}{B(s)} \Upsilon_{\alpha}^{*}(s)+\frac{\Upsilon_{\alpha}(0)\left(\lambda \bar{F}^{*}(s)+\frac{\alpha}{s}\right)+\frac{\sigma^{2}}{2} \Upsilon_{\alpha}^{\prime}(0)-R^{*}(s)-\alpha P^{*}(s)}{B(s)}=0$

we find for $\delta>0$ and $s>0$ that:

$\Upsilon_{\alpha}^{*}(s)=\int_{s}^{\infty}\left[\frac{\Upsilon_{\alpha}(0)\left(\lambda \bar{F}^{*}(x)+\frac{\alpha}{x}\right)+\frac{\sigma^{2}}{2} \Upsilon_{\alpha}^{\prime}(0)-R^{*}(x)-\alpha P^{*}(x)}{B(x)}\right] E_{\alpha}(s, x) d x$

where

$$
E_{\alpha}(s, x)=e^{\int_{s}^{x} \frac{\lambda \bar{F}^{*}(z)-A(z)}{B(z)}}=e^{\int_{s}^{x} \frac{\lambda \bar{F}^{*}(z)-\left(c+\frac{\sigma^{2} z}{2}-\frac{\sigma_{r}^{2}}{2}-\frac{\alpha}{z}\right)}{B(z)}} d z
$$

Before simplifying further this solution, we analyze the behavior of the integrating factor $E_{\alpha}(s, x)$. We note by using the decomposition

$$
\begin{aligned}
-\frac{c+\frac{\sigma^{2} z}{2}-\frac{\sigma_{r}^{2}}{2}-\frac{\alpha}{z}}{\delta+\frac{\sigma_{r}^{2}}{2} z}= & -\frac{\sigma^{2}}{\sigma_{r}^{2}}+\frac{\alpha}{\delta z}+\frac{-c+\delta\left(\sigma / \sigma_{r}\right)^{2}+\left(1-\frac{\alpha}{\delta}\right) \frac{\sigma_{r}^{2}}{2}}{\delta+\frac{\sigma_{r}^{2}}{2} z}= \\
& -\frac{\sigma^{2}}{\sigma_{r}^{2}}+\frac{\alpha}{\delta z}+\frac{\left(\rho+1-\frac{\alpha}{\delta}\right) \frac{\sigma_{r}^{2}}{2}}{\delta+\frac{\sigma_{r}^{2}}{2} z}
\end{aligned}
$$

where $\rho=\frac{2\left(\delta \sigma^{2}-\sigma_{r}^{2} c\right)}{\sigma_{r}^{4}}$, that

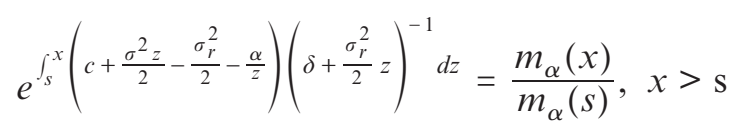

and

$$
m_{\alpha}(x)= \begin{cases}e^{-\left(\frac{\sigma^{2}}{\sigma_{r}^{2}}\right) x} B(x)^{1+\rho-\frac{\alpha}{\delta}} x^{\frac{\alpha}{\delta}} & \text { for } \sigma_{r}^{2}>0 \\ x^{\frac{\alpha}{\delta}} e^{-\frac{1}{\delta}\left[\left(\frac{\sigma^{2}}{4}\right) x^{2}+c x\right]} & \text { for } \sigma_{r}^{2}=0\end{cases}
$$

In conclusion, the integrating factor $E_{\alpha}(s, x)$ may be written as

$$
E_{\alpha}(s, x)=\frac{m_{\alpha}(x)}{m_{\alpha}(s)} e^{\lambda \int_{s}^{x} \frac{\bar{F}^{*}(z)}{B(z)} d z}
$$


Lemma 2. For $\alpha>0$ and $\delta>0$, it holds that:

$$
m_{\alpha}(0)=0,
$$

in both cases described in (12) and

$$
m_{0}(0)=\lim _{s \rightarrow 0^{+}} m_{0}(s)= \begin{cases}\delta^{\rho+1} & \text { for } \sigma_{r}^{2}>0 \\ 1 & \text { for } \sigma_{r}^{2}=0\end{cases}
$$

Note: This obvious result will be important later since $m_{\alpha}(s)$ appears in the denominator of the Laplace transform then, ensuring the analyticity of our Laplace transform at $s=0$ despite the pole introduced by $m_{\alpha}(s)$, will provide us with a second equation necessary to determine the usual extra boundary unknowns appearing in the equation determining the Laplace transform.

Lemma 3. Suppose that $s>0, c>0, \sigma \geq 0, \sigma_{r} \geq 0$, and assume also that $c>\frac{\sigma_{r}^{2}}{2}$ when $\sigma=0$ and $\sigma_{r}>0$. Then, the integrating factor $E_{\alpha}(s, x)$ defined in (11) has for any $\varepsilon$ the order of magnitude $E_{\alpha}(s, x)=o(H(x))$ when $x \rightarrow \infty$, with $H(x)$ given by:

$$
H(x)= \begin{cases}e^{-\left(\frac{\sigma^{2}}{\sigma_{r}^{2}}-\varepsilon\right) x} & \text { for } \sigma_{r}>0, \sigma>0 \\ \left(\delta+\frac{\sigma_{r}^{2}}{2} x\right)^{\rho+1+\varepsilon} & \text { for } \sigma_{r}>0, \sigma=0 \\ e^{-\frac{1}{\delta}\left(\frac{\sigma^{2}-\varepsilon}{4}\right) x^{2}} & \text { for } \sigma_{r}=0, \sigma>0 \\ e^{-\frac{1}{\delta}(c-\varepsilon) x} & \text { for } \sigma_{r}=0, \sigma=0\end{cases}
$$

and $\lim _{x \rightarrow \infty} E_{\alpha}(s, x)=0$.

Proof: We note first that $e^{\lambda \int_{s}^{x} \frac{\bar{F}^{*}(z)}{B(z)} d z}$ is bounded above by a power. Indeed, since $\bar{F}(x)$ is a non-increasing function and $\bar{F}(0)=1$, we have $\bar{F}^{*}(z)=\int_{0}^{\infty} e^{-z x} \bar{F}(x) d x<$ $\frac{1}{z}$ and

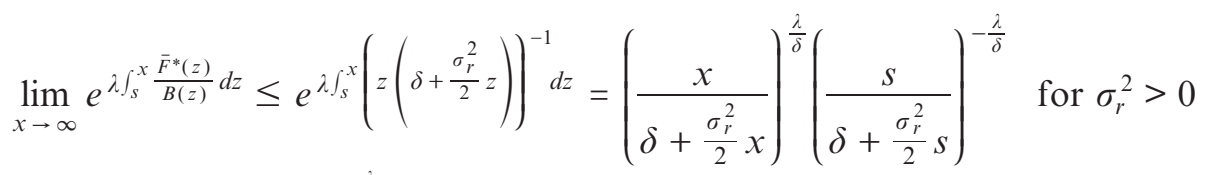

$$
\begin{aligned}
& \leq\left(\frac{x}{s}\right)^{\frac{\lambda}{\delta}} \\
& \text { for } \sigma_{r}^{2}=0
\end{aligned}
$$


Plugging this in (13), we find the bounds

$$
\begin{array}{rlrl}
E_{\alpha}(s, x) & \leq \frac{\left(\frac{\sigma_{r}^{2}}{2}\right)^{-\frac{\alpha+\lambda}{\delta}}}{m_{\alpha}(s)}\left(\frac{s}{\delta+\frac{\sigma_{r}^{2}}{2} s}\right)^{-\frac{\lambda}{\delta}} e^{-\left(\frac{\sigma^{2}}{\sigma_{r}^{2}}\right) x\left(\delta+\frac{\sigma_{r}^{2}}{2} x\right)^{\rho+1}} & & \text { for } \sigma_{r}^{2}>0 \\
& \leq \frac{s^{-\frac{\lambda}{\delta}}}{m_{\alpha}(s)} e^{-\frac{1}{\delta}\left[\left(\frac{\sigma^{2}}{4}\right) x^{2}+c x\right] x^{\frac{\alpha+\lambda}{\delta}}} & \text { for } \sigma_{r}^{2}=0
\end{array}
$$

which yield the order of magnitude. The limit follows since the function $E_{\alpha}(s, x)$ is positive; the presence of the exponential damping implies that limit is always zero for $\sigma^{2}>0$, and the same holds when $\sigma^{2}=0$, if $\rho<-1 \Leftrightarrow c>\frac{\sigma_{r}^{2}}{2}$

Notes:

1) The previous work by Sundt \& Teugels (1995) (who assumed $\sigma=\sigma_{r}=0$ ) also made use of the assumption $c>0$ (see the end of section 2).

2) In the model without jumps $(\sigma=\lambda=0)$, the condition $c>\frac{\sigma_{r}^{2}}{2}$ makes the boundary $u=0$ unattainable.

\section{THE MAIN RESULT}

Theorem 2. a) For any $\alpha \geq 0, \delta>0, c>0, s>0$ and assuming $c>\frac{\sigma_{r}^{2}}{2}$ in the particular case $\sigma=0, \sigma_{r}>0$, the Laplace transform $\Upsilon_{\alpha}^{*}(s)=\int_{0}^{\infty} e^{-s u} d_{u} Y_{\alpha}^{2}(u)$ satisfies:

$$
\begin{aligned}
\Upsilon_{\alpha}^{*}(s)= & \frac{e^{\lambda \int_{0}^{s} \frac{\bar{F}^{*}(z)}{B(z)} d z}}{m_{\alpha}(s)}\left(\varsigma+\int_{0}^{s}\left(R^{*}(x)+\alpha P^{*}(x)-\beta_{1}-\beta_{2} x\right) e^{\lambda \int_{0}^{x} \frac{\bar{F}^{*}(z)}{B(z)} d z} \frac{m_{\alpha}(x)}{B(x)} d x\right) \\
& -\Upsilon_{\alpha}(0)
\end{aligned}
$$

where

$$
\begin{aligned}
& \beta_{1}:=\Upsilon_{\alpha}(0)\left(c-\frac{\sigma_{r}^{2}}{2}\right)+\frac{\sigma^{2}}{2} \Upsilon_{\alpha}^{\prime}(0), \quad \beta_{2}:=\Upsilon_{\alpha}(0) \frac{\sigma^{2}}{2}, B(x):=\delta+\frac{\sigma_{r}^{2}}{2} x
\end{aligned}
$$

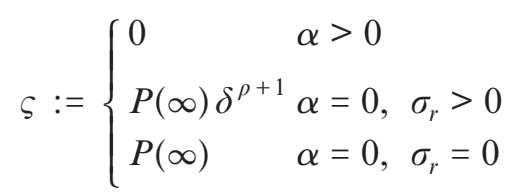

and $m_{\alpha}(x)$ is given by (12). 
b) For $\sigma>0, \Upsilon_{\alpha}(0)=\pi\left(0_{-}\right)$and

$$
\Upsilon_{\alpha}^{\prime}(0)=\frac{\varsigma+\int_{0}^{\infty}\left(R^{*}(x)+\alpha P^{*}(x)-\pi\left(0_{-}\right)\left(c-\frac{\sigma_{r}^{2}}{2}-\frac{\sigma^{2}}{2} x\right)\right) e^{\lambda \int_{0}^{x} \frac{\bar{F}^{*}(z)}{B(z)} d z \frac{m_{\alpha}(x)}{B(x)} d x}}{\frac{\sigma^{2}}{2} \int_{0}^{\infty} e^{\lambda \int_{0}^{x} \frac{\bar{F}^{*}(z)}{B(z)} d z} \frac{m_{\alpha}(x)}{B(x)} d x}
$$

c) For $\sigma=0$

$$
\begin{aligned}
\Upsilon_{\alpha}(0) & =\frac{\varsigma+\int_{0}^{\infty}\left(R^{*}(x)+\alpha P^{*}(x)\right) e^{\lambda \int_{0}^{x} \frac{\bar{F}^{*}(z)}{B(z)} d z} \frac{m_{\alpha}(x)}{B(x)} d x}{\left(c-\frac{\sigma_{r}^{2}}{2}\right) \int_{0}^{\infty} e^{\lambda \int_{0}^{F^{*}} \frac{\bar{F}^{*}(z)}{B(z)} d z \frac{m_{\alpha}(x)}{B(x)} d x}} \\
& = \begin{cases}\frac{\varsigma+\int_{0}^{\infty}\left(R^{*}(x)+\alpha P^{*}(x)\right) x^{\frac{\alpha}{\delta}}(B(x))^{\rho-\frac{\alpha}{\delta}} e^{\int_{0}^{x} \frac{\lambda \bar{F}^{*}(z)}{B(z)} d z} d x}{\left(c-\frac{\sigma_{r}^{2}}{2}\right) \int_{0}^{\infty} x^{\frac{\alpha}{\delta}}(B(x))^{\rho-\frac{\alpha}{\delta}} e^{\int_{0}^{x} \frac{\lambda \bar{F}^{*}(z)}{B(z)} d z} d x} & \text { when } \sigma_{r}^{2}>0 \\
\frac{\varsigma+\delta^{-1} \int_{0}^{\infty}\left(R^{*}(x)+\alpha P^{*}(x)\right) x^{\frac{\alpha}{\delta}} e^{-\frac{c}{\delta} x} e^{\frac{\lambda}{\delta} \int_{0}^{x} \bar{F}^{*}(z) d z} d x}{\frac{c}{\delta} \int_{0}^{\infty} x^{\frac{\alpha}{\delta}} e^{-\frac{c}{\delta} x} e^{\frac{\lambda}{\delta} \int_{0}^{x} \bar{F}^{*}(z) d z} d x} & \text { when } \sigma_{r}^{2}=0\end{cases}
\end{aligned}
$$

where $\rho=\frac{2\left(\delta \sigma^{2}-\sigma_{r}^{2} c\right)}{\sigma_{r}^{4}}$.

Proof: Simplifying further the equation (10)

$$
\begin{aligned}
& \Upsilon_{\alpha}^{*}(s)=\int_{s}^{\infty}\left[\frac{\Upsilon_{\alpha}(0)\left(\lambda \bar{F}^{*}(x)+\frac{\alpha}{x}\right)+\frac{\sigma^{2}}{2} \Upsilon_{\alpha}^{\prime}(0)-R^{*}(x)-\alpha P^{*}(x)}{B(x)}\right] E_{\alpha}(s, x) d x \\
& =\Upsilon_{\alpha}(0) \int_{s}^{\infty}\left[\frac{\lambda \bar{F}^{*}(x)+\frac{\alpha}{x}-\left(c+\frac{\sigma^{2} x}{2}-\frac{\sigma_{r}^{2}}{2}\right)+\left(c+\frac{\sigma^{2} x}{2}-\frac{\sigma_{r}^{2}}{2}\right)}{B(x)}\right] E_{\alpha}(s, x) d x \\
& +\int_{s}^{\infty}\left(\frac{\frac{\sigma^{2}}{2} \Upsilon_{\alpha}^{\prime}(0)-R^{*}(x)-\alpha P^{*}(x)}{B(x)}\right) E_{\alpha}(s, x) d x=\Upsilon_{\alpha}(0)\left[E_{\alpha}(s, x)\right]_{x=s}^{\infty} \\
& +\int_{s}^{\infty}\left(\frac{\Upsilon_{\alpha}(0)\left(c+\frac{\sigma^{2} x}{2}-\frac{\sigma_{r}^{2}}{2}\right)+\frac{\sigma^{2}}{2} \Upsilon_{\alpha}^{\prime}(0)-R^{*}(x)-\alpha P^{*}(x)}{B(x)}\right) E_{\alpha}(s, x) d x
\end{aligned}
$$




$$
=\int_{s}^{\infty}\left(\frac{\Upsilon_{\alpha}(0)\left(c+\frac{\sigma^{2} x}{2}-\frac{\sigma_{r}^{2}}{2}\right)+\frac{\sigma^{2}}{2} \Upsilon_{\alpha}^{\prime}(0)-R^{*}(x)-\alpha P^{*}(x)}{B(x)}\right) E_{\alpha}(s, x)-\Upsilon_{\alpha}(0)
$$

since $\lim _{x \rightarrow \infty} E_{\alpha}(s, x)=0$ (see Lemma 3 and (13)), we find

$$
\begin{aligned}
&\left(\Upsilon_{\alpha}^{*}(s)+\Upsilon_{\alpha}(0)\right) m_{\alpha}(s)=\int_{s}^{\infty}\left(\Upsilon_{\alpha}(0)\left(c-\frac{\sigma_{r}^{2}}{2}\right)+\frac{\sigma^{2}}{2} \Upsilon_{\alpha}^{\prime}(0)+\right. \\
&\left.\Upsilon_{\alpha}(0) \frac{\sigma^{2}}{2} x-R^{*}(x)-\alpha P^{*}(x)\right) e^{\lambda \int_{s}^{x} \frac{\bar{F}^{*}(z)}{B(z)} d z} \frac{m_{\alpha}(x)}{B(x)} d x
\end{aligned}
$$

Note that the integrals above are well defined by the bounds obtained in Lemma 3. For example, when $\sigma=0, \sigma_{r}>0$, the convergence is assured by the fact that $E_{\alpha}(s, x)$ may be bounded by a power with exponent which may be made smaller than -1 for $\varepsilon$ small enough, and that the remaining fraction which multiplies it may be bounded by a constant.

Recall now that

$$
\lim _{s \rightarrow 0^{+}} \Upsilon_{\alpha}^{*}(s)+\Upsilon_{\alpha}(0)=\lim _{u \rightarrow \infty} \Upsilon_{\alpha}(0)=\lim _{u \rightarrow \infty} P(u)<\infty
$$

because $P(u)$ is bounded and from Lemma 2

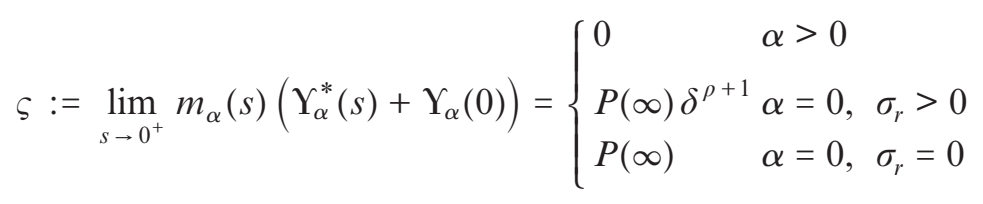

and finally

$$
\begin{aligned}
\varsigma & =\Upsilon_{\alpha}(0) \int_{0}^{\infty}\left(\left(c-\frac{\sigma_{r}^{2}}{2}\right)+\frac{\sigma^{2}}{2} x\right) e^{\lambda \int_{0}^{x} \frac{\bar{F}^{*}(z)}{B(z)} d z} \frac{m_{\alpha}(x)}{B(x)} d x \\
& +\frac{\sigma^{2}}{2} \Upsilon_{\alpha}^{\prime}(0) \int_{0}^{\infty} e^{\lambda \int_{0}^{x} \frac{\bar{F}^{*}(z)}{B(z)} d z} \frac{m_{\alpha}(x)}{B(x)} d x \\
& -\int_{0}^{\infty}\left(R^{*}(x)+\alpha P^{*}(x)\right) e^{\lambda \int_{0}^{x} \frac{\bar{F}^{*}(z)}{B(z)} d z} \frac{m_{\alpha}(x)}{B(x)} d x
\end{aligned}
$$

This yields respectively the two expressions b) and c). 
Using the last expression, clearly

$$
\begin{aligned}
\int_{s}^{\infty} & \left(\beta_{1}+\beta_{2} x-R^{*}(x)-\alpha P^{*}(x)\right) e^{\lambda \int_{s}^{x} \frac{\bar{F}^{*}(z)}{B(z)} d z} \frac{m_{\alpha}(x)}{B(x)} d x \\
& =e^{-\lambda \int_{0}^{s} \frac{\bar{F}^{*}(z)}{B(z)} d z} \int_{s}^{\infty}\left(\beta_{1}+\beta_{2} x-R^{*}(x)-\alpha P^{*}(x)\right) e^{\lambda \int_{0}^{x} \frac{\bar{F}^{*}(z)}{B(z)} d z} \frac{m_{\alpha}(x)}{B(x)} d x \\
& =e^{-\lambda \int_{0}^{s} \frac{\bar{F}^{*}(z)}{B(z)} d z}\left(\varsigma+\int_{0}^{s}\left(R^{*}(x)+\alpha P^{*}(x)-\beta_{1}-\beta_{2} x\right) e^{\lambda \int_{0}^{x} \frac{\bar{F}^{*}(z)}{B(z)} d z} \frac{m_{\alpha}(x)}{B(x)} d x\right)
\end{aligned}
$$

and (14) can be given by

$$
\begin{aligned}
\Upsilon_{\alpha}^{*}(s)= & \frac{e^{-\lambda \int_{0}^{s} \frac{\bar{F}^{*}(z)}{B(z)} d z}}{m_{\alpha}(s)}\left(\varsigma+\int_{0}^{s}\left(R^{*}(x)+\alpha P^{*}(x)-\beta_{1}-\beta_{2} x\right) e^{\lambda \int_{0}^{x} \frac{\bar{F}^{*}(z)}{B(z)} d z} \frac{m_{\alpha}(x)}{B(x)} d x\right) \\
& -\Upsilon_{\alpha}(0)
\end{aligned}
$$

\section{EXAMPLES}

Some previous results in literature are now revisited.

\subsection{The Gerber-Shiu penalty function}

With surplus $u=0$, the Laplace transform in time has been called sometimes the Gerber-Shiu penalty function, see Gerber and Shiu (1998) and Willmot and Dickson (2003)

$$
\Upsilon_{\alpha}(0)=\int_{0}^{\infty} \alpha e^{-\alpha t} v_{t}(0) d t
$$

\subsection{The expected penalty at ruin}

Note that the expected deficit at ruin is also an easy corollary, the penalty at ruin being $\pi(-u)=u$ thus

$$
\begin{aligned}
& R(u)=\lambda \int_{u}^{\infty} \pi(u-x) f(x) d x=\lambda \int_{u}^{\infty}(x-u) \mu e^{-\mu x} d x=\frac{\lambda}{\mu} e^{-\mu x} \\
& R^{*}(x)=\frac{\lambda}{\mu(\mu+z)}
\end{aligned}
$$

When a deterministic rate of interest is assumed, $\sigma_{r}^{2}=0$, and $\sigma^{2}=0$, Cai and Dickson (2002) obtained the expected penalty at ruin for an infinite time 
horizon. Their expression 3.8 (mind the change in the notation) coincides with our Theorem 2.c)

$$
v(0)=\frac{\int_{0}^{\infty} R^{*}(x) e^{-\frac{c}{\delta} x} e^{\frac{\lambda}{\delta} \int_{0}^{x} \bar{F}^{*}(z) d z} d x}{c \int_{0}^{\infty} e^{-\frac{c}{\delta} x} e^{\frac{\lambda}{\delta} \int_{0}^{x} \bar{F}^{*}(z) d z} d x}
$$

\subsection{The survival probability}

The survival probability, $\Phi_{\alpha}(0)$, with zero initial reserves can be obtained using Theorem 2 where $P(u)=1$ and $\pi(-u)=0, u>0$. Since when $\sigma^{2}>0$ the presence of brownian motion implies trivially $\Phi(0)=0$, thus we assume $\sigma^{2}=0$. For $\alpha>0$

$$
\Phi_{\alpha}(0)= \begin{cases}\frac{\alpha \int_{0}^{\infty} x^{\frac{\alpha}{\delta}-1}(B(x))^{\rho-\frac{\alpha}{\delta}} e^{\lambda \int_{0}^{x} \frac{\bar{F}^{*}(z)}{B(z)} d z} d x}{\left(c-\frac{\sigma_{r}^{2}}{2}\right) \int_{0}^{\infty} x^{\frac{\alpha}{\delta}}(B(x))^{\rho-\frac{\alpha}{\delta}} e^{\lambda \int_{0}^{x} \frac{\bar{F}^{*}(z)}{B(z)} d z} d x}, & \text { when } \sigma_{r}^{2}>0 \\ \left(\frac{\alpha}{c}\right) \frac{\int_{0}^{\infty} x^{\frac{\alpha}{\delta}-1} e^{-\frac{c}{\delta} x} e^{\frac{\lambda}{\delta} \int_{0}^{x} \bar{F}^{*}(z) d z} d x}{\int_{0}^{\infty} x^{\frac{\alpha}{\delta}} e^{-\frac{c}{\delta} x} e^{\frac{\lambda}{\delta} \int_{0}^{x} \bar{F}^{*}(z) d z} d x}, & \text { when } \sigma_{r}^{2}=0\end{cases}
$$

In the ultimate case, we find

$$
\Phi_{\alpha}(0)= \begin{cases}\frac{c-\frac{\sigma_{r}^{2}}{2}}{\delta^{\rho+1}} \int_{0}^{\infty}(B(x))^{\rho} e^{\lambda \int_{0}^{x} \frac{\bar{F}^{*}(z)}{B(z)} d z} d x & \text { when } \sigma_{r}^{2}>0 \\ \left(\frac{c}{\delta}\right) \int_{0}^{\infty} e^{-\frac{c}{\delta} x} e^{\frac{\lambda}{\delta} \int_{0}^{x} \bar{F}^{*}(z) d z} d x & \text { when } \sigma_{r}^{2}=0\end{cases}
$$

The last expression coincides with equation 14 in Sundt and Teugels (1995). We further consider the ultimate case when $\sigma_{r}^{2}=0$ using the expression for $\Phi^{*}(s)=\int_{0}^{\infty} e^{-s u} d_{u} \Phi(u)$ provided in Theorem 2, we find

$$
\begin{aligned}
\Phi^{*}(s) & =e^{\frac{c}{\delta} s-\frac{\lambda}{\delta} \int_{0}^{s} \bar{F}^{*}(z) d z}\left(1-\Phi(0)\left(\frac{c}{\delta}\right) \int_{0}^{s} e^{\frac{\lambda}{\delta} \int_{0}^{x} \bar{F}^{*}(z) d z} e^{-\frac{c}{\delta} x} d x\right)-\Phi(0) \\
& =\Phi(0)\left(\frac{c}{\delta}\right) \int_{s}^{\infty} e^{-\frac{c}{\delta}(x-s)} e^{\frac{\lambda}{\delta} \int_{0}^{x} \bar{F}^{*}(z) d z} d x-\Phi(0)
\end{aligned}
$$

a result previously offered by expression (12) in Sund and Teugels (1995). The Segerdahl's formula follows easily from the previous result as proved in the mentioned reference. 


\subsection{The ruin probability for exponential claims}

Let us now assume that the claims are exponential

$$
\begin{aligned}
& f(z)=\mu e^{-\mu z} \quad z>0 \\
& \bar{F}^{*}(x)=(\mu+x)^{-1}
\end{aligned}
$$

and

$$
e^{\lambda \int_{0}^{x} \frac{\bar{F}^{*}(z)}{B(z)} d z}= \begin{cases}e^{\frac{\lambda}{\delta} \int_{0}^{x} \bar{F}^{*}(z) d z}=\left(\frac{\mu+x}{\mu}\right)^{\frac{\lambda}{\delta}} & \text { if } \sigma_{r}=0 \\ e^{\lambda \int_{0}^{x}(\mu+z)^{-1}\left(\delta+\frac{\sigma_{r}^{2}}{2} z\right)^{-1} d z}=\left(\frac{\mu}{\delta}\right)^{\beta}\left(\frac{\delta+\frac{\sigma_{r}^{2}}{2} x}{\mu+x}\right)^{\beta} & \text { else }\end{cases}
$$

where $\beta=\lambda\left(\frac{\sigma_{r}^{2}}{2} \mu-\delta\right)^{-1}$. We will provide below the probabilities of killed ruin $(P(u)=0$ and $\pi(-u)=1, u>0)$

$$
R^{*}(x)=\frac{\lambda}{\mu+x}, P^{*}(x)=0
$$

when $\sigma^{2}=0$.

1. $\sigma_{r}^{2}>0$, see Theorem 2

$$
\begin{aligned}
\psi_{\alpha}(0) & =\frac{\frac{\lambda}{\mu} \int_{0}^{\infty}(x+\mu)^{-(\beta-1)} x^{\frac{\alpha}{\delta}}\left(x+\frac{2 \delta}{\sigma_{r}^{2}}\right)^{-\left(\frac{\alpha}{\delta}-\rho-\beta\right)} d x}{\left(c-\frac{\sigma_{r}^{2}}{2}\right) \int_{0}^{\infty}(x+\mu)^{-(\beta-1)} x^{\frac{\alpha}{\delta}}\left(x+\frac{2 \delta}{\sigma_{r}^{2}}\right)^{-\left(\frac{\alpha}{\delta}-\rho-\beta\right)} d x} \\
& =\frac{\frac{2 \delta \lambda}{\sigma_{r}^{2}}}{\left(c-\frac{\sigma_{r}^{2}}{2}\right) \mu^{2}} \frac{\operatorname{Beta}\left(\frac{\alpha}{\delta}+1,-\rho\right) F\left(\beta+1, \frac{\alpha}{\delta}+1 ; \frac{\alpha}{\delta}+1-\rho ; 1-\frac{2 \delta}{\mu \sigma_{r}^{2}}\right)}{\operatorname{Beta}\left(\frac{\alpha}{\delta}+1,-\rho-1\right) F\left(\beta, \frac{\alpha}{\delta}+1 ; \frac{\alpha}{\delta}-\rho ; 1-\frac{2 \delta}{\mu \sigma_{r}^{2}}\right)} \\
& =\left(c-\frac{\sigma_{r}^{2}}{2}\right)^{-1} \mu^{-2}\left(\frac{2 \delta \lambda}{\sigma_{r}^{2}}\right)\left(\frac{\rho+1}{\rho-\frac{\alpha}{\delta}}\right) \frac{F\left(\beta+1, \frac{\alpha}{\delta}+1 ; \frac{\alpha}{\delta}+1-\rho ; 1-\frac{2 \delta}{\mu \sigma_{r}^{2}}\right)}{F\left(\beta, \frac{\alpha}{\delta}+1 ; \frac{\alpha}{\delta}-\rho ; 1-\frac{2 \delta}{\mu \sigma_{r}^{2}}\right)}
\end{aligned}
$$

also mind that $\rho=-\frac{2 c}{\sigma_{r}^{2}}<-1$ and 


$$
\begin{aligned}
& \int_{0}^{\infty}(x+\mu)^{-(\beta+1)} x^{\left(\frac{\alpha}{\delta}+1\right)^{-1}}\left(x+\frac{2 \delta}{\sigma_{r}^{2}}\right)^{-\left(\frac{\alpha}{\delta}-\rho-\beta\right)} d x \\
& =\mu^{-(\beta+1)}\left(\frac{2 \delta}{\sigma_{r}^{2}}\right)^{\rho+(\beta+1)} \operatorname{Beta}\left(\frac{\alpha}{\delta}+1,-\rho\right) F\left(\beta+1, \frac{\alpha}{\delta}+1 ; \frac{\alpha}{\delta}+1-\rho ; 1-\frac{2 \delta}{\mu \sigma_{r}^{2}}\right)
\end{aligned}
$$

see expression 3.197.1 in Gradshteyn \& Ryzhik (1994) where $F(\cdot)$ is the Gauss's hypergeometric function. The Laplace transform is also available, in terms of Appell hypergeometric functions.

2. $\sigma_{r}^{2}=0$

$$
\psi_{\alpha}(0)=\frac{\frac{\lambda}{\mu} \int_{0}^{\infty}(\mu+x)^{\frac{\lambda}{\delta}-1} x^{\frac{\alpha}{\delta}} e^{-\frac{c}{\delta} x} d x}{c \int_{0}^{\infty}(\mu+x)^{\frac{\lambda}{\delta}-1} x^{\frac{\alpha}{\delta}} e^{-\frac{c}{\delta} x} d x}
$$

we can get, see also Paulsen \& Gjessing (1997), example 2.1

$$
\begin{aligned}
\psi_{\alpha}(0) & =\left(\frac{\lambda}{c \mu}\right) \frac{\int_{0}^{\infty} x^{\frac{\alpha}{\delta}} e^{-\frac{c}{\delta} x}(\mu+x)^{\frac{\lambda}{\delta}-1} d x}{\int_{0}^{\infty} x^{\frac{\alpha}{\delta}} e^{-\frac{c}{\delta} x}(\mu+x)^{\frac{\lambda}{\delta}} d x}=\left(\frac{\lambda}{c \mu}\right) \frac{\int_{0}^{\infty} x^{\frac{\alpha}{\delta}} e^{-\frac{c \mu}{\delta} x}(1+x)^{\frac{\lambda}{\delta}-1} d x}{\int_{0}^{\infty} x^{\frac{\alpha}{\delta}} e^{-\frac{c \mu}{\delta} x}(1+x)^{\frac{\lambda}{\delta}} d x} \\
& =\left(\frac{\lambda}{c \mu}\right) \frac{U\left(1+\frac{\alpha}{\delta}, 1+\frac{\alpha+\lambda}{\delta}, \frac{c \mu}{\delta}\right)}{U\left(1+\frac{\alpha}{\delta}, 2+\frac{\alpha+\lambda}{\delta}, \frac{c \mu}{\delta}\right)}
\end{aligned}
$$

where

$$
U(a, b, c)=\Gamma(a)^{-1} \int_{0}^{\infty} t^{a-1} e^{-c t}(1+t)^{b-a-1} d t
$$

is the confluent Tricomi hypergeometric function and

$$
\begin{aligned}
U\left(1+\frac{\alpha}{\delta}, 2+\frac{\alpha+\lambda}{\delta}, \frac{c \mu}{\delta}\right) & =U\left(1+\frac{\alpha}{\delta}, 1+\frac{\alpha+\lambda}{\delta}, \frac{c \mu}{\delta}\right) \\
& +\left(1+\frac{\alpha}{\delta}\right) U\left(2+\frac{\alpha}{\delta}, 2+\frac{\alpha+\lambda}{\delta}, \frac{c \mu}{\delta}\right)
\end{aligned}
$$

see expression 13.4.7 in Abramowitz and Stegun (1970). 


\section{APPENDIX}

The same thread of Theorem 5 in Gaier \& Grandits (2005) shall be followed in order to prove the existence of a solution of (7). Thus defining $y(u):=\Upsilon_{\alpha}^{\prime}(u)$ we will prove that $y \in C^{1}[0, \infty) \cap L^{1} \cap L^{\infty}$ and thus $\Upsilon_{\alpha} \in C^{2}[0, \infty)$. Assuming $\bar{F}(0)=1$ we can write

$$
\begin{aligned}
& (c+d u) y(u)+\left(\frac{\sigma^{2}+\sigma_{r}^{2} u}{2}\right) y^{\prime}(u) \\
& =\Upsilon_{\alpha}(0)(\lambda \bar{F}(u)+\alpha)+\int_{0}^{u} y(u-x)(\lambda \bar{F}(x)+\alpha) d x+R(u)+\alpha P(u)
\end{aligned}
$$

with initial condition

$$
y(0)= \begin{cases}\frac{(\lambda+\alpha) \Upsilon_{\alpha}(0)+R(0)+\alpha P(0)}{c}, & \sigma=0 \\ \frac{(\lambda+\alpha) \pi\left(0^{-}\right)-\frac{\sigma^{2}}{2} \Upsilon_{\alpha}^{\prime \prime}(0)+R(0)+\alpha P(0)}{c}, & \sigma>0\end{cases}
$$

Let us now define

$$
\varphi^{(y)}(u):=\Upsilon_{\alpha}(0)(\lambda \bar{F}(u)+\alpha)+\int_{0}^{u} y(u-x)(\lambda \bar{F}(x)+\alpha) d x+R(u)+\alpha P(u)
$$

where

$$
R(u)=\lambda \int_{0}^{\infty} \pi(x) f(x+u) d x
$$

and finally

$$
(c+\delta u) y(u)+\left(\frac{\sigma^{2}+\sigma_{r}^{2} u}{2}\right) y^{\prime}(u)=\varphi^{(y)}(u)
$$

The solution for $u \geq 0$ can be given by

$$
y(u)=\left(\int_{0}^{u} \varphi^{(y)}(z) e^{z m_{1}}(D(z))^{\left(m_{2}-1\right)} d z\right) e^{-u m_{1}}(D(u))^{-m_{2}}+1_{\{\sigma>0\}} y(0)
$$

where for convenience 


$$
m_{1}=\frac{2 \delta}{\sigma_{r}^{2}} ; \quad m_{2}=\frac{2\left(c \sigma_{r}^{2}-\delta \sigma^{2}\right)}{\sigma_{r}^{4}} ; \quad D(z)=\frac{\sigma^{2}+\sigma_{r}^{2} z}{2}
$$

Let us start proving the following lemma,

Lemma 4. For a function $h(z) \in C\left[0, u_{0}\right]$ for small $u_{0}$

$$
\begin{aligned}
\left(\int_{0}^{u} h(z) e^{z m_{1}}(D(z))^{\left(m_{2}-1\right)} d z\right) e^{-u m_{1}}(D(u))^{-m_{2}} & \\
= & \begin{cases}\frac{(\lambda+\alpha) \Upsilon_{\alpha}(0)+R(0)+\alpha P(0)}{c}, & \sigma=0 \\
\frac{(\lambda+\alpha) \pi\left(0^{-}\right)-\frac{\sigma^{2}}{2} \Upsilon_{\alpha}^{\prime \prime}(0)+R(0)+\alpha P(0)}{c}, & \sigma>0\end{cases}
\end{aligned}
$$

Proof: Let us define

$$
\begin{aligned}
& M(u)=\int_{0}^{u} h(z) e^{z m_{1}}(D(z))^{\left(m_{2}-1\right)} d z, N(u)=e^{-u m_{1}}(D(u))^{-m_{2}} \\
& M^{\prime}(u)=\frac{h(u)}{N(u) D(u)}, \quad N^{\prime}(u)=N(u)(-1)\left(m_{1}+\frac{m_{2}}{D(u)} \frac{\sigma_{r}^{2}}{2}\right)
\end{aligned}
$$

and the Taylor expansion for small $u$ yields

$$
\left(\lim _{u \rightarrow 0^{+}}[M(u) N(u)]\right)+\left(\lim _{u \rightarrow 0^{+}}\left[\frac{h(u)}{D(u)}-M(u) N(u)\left(m_{1}+\frac{m_{2}}{D(u)} \frac{\sigma_{r}^{2}}{2}\right)\right]\right) u+o(u)
$$

For $\sigma=0, m_{2}=\frac{2 c}{\sigma_{r}^{2}}$ and using L'Hopital's rule

$$
\begin{aligned}
\lim _{u \rightarrow 0^{+}} M(u) N(u) & =\left(\int_{0}^{u} h(z) e^{z m_{1}}(D(z))^{m_{2}-1} d z\right) e^{-u m_{1}}(D(u))^{-m_{2}} \\
& =\lim _{u \rightarrow 0^{+}} \frac{h(u)}{m_{1} D(u)+c}=\frac{h(0)}{c}
\end{aligned}
$$

and

$$
\begin{aligned}
\lim _{u \rightarrow 0^{+}}\left[\frac{h(u)}{D(u)}-\right. & \left.M(u) N(u)\left(m_{1}+\frac{c}{D(u)}\right)\right] \\
& =\lim _{u \rightarrow 0^{+}}\left[\frac{h(u)}{D(u)}-\frac{h(u)}{m_{1} D(u)+c}\left(m_{1}+\frac{c}{D(u)}\right)\right]
\end{aligned}
$$




$$
\begin{aligned}
& =\lim _{u \rightarrow 0^{+}}\left[\frac{h(u)}{D(u)}\left(1-\frac{c}{m_{1} D(u)+c}\right)-\frac{h(u) m_{1}}{m_{1} D(u)+c}\right] \\
& =\lim _{u \rightarrow 0^{+}}\left[\frac{h(u)}{D(u)}\left(\frac{m_{1} D(u)}{m_{1} D(u)+c}\right)-\frac{h(u) m_{1}}{m_{1} D(u)+c}\right]=0
\end{aligned}
$$

The result for $\sigma>0$ is straightforward.

Step 1. The integral equation above has a solution $y \in C\left[0, u_{0}\right]$ for $u_{0}$ small enough, using Banach's Fixed Point Theorem. The operator

$T y(u)=\left(\int_{0}^{u} \varphi^{(y)}(z) e^{z m_{1}}(D(z))^{\left(m_{2}-1\right)} d z\right) e^{-u m_{1}}(D(u))^{-m_{2}}+1_{\{\sigma>0\}} y(0), u \geq 0$

is a selfmapping contraction on the Banach space

$$
x:=\left\{y \in C\left[0, u_{0}\right], y(0)\right\}
$$

for $u_{0}$ small enough. Using the lemma above for $h(u):=\varphi^{(y)}(u)$

$$
\lim _{x \downarrow 0} T y(u)=y(0)
$$

the selfmapping proof unfolds. Now for $y_{1}, y_{2} \in x$

$$
\begin{aligned}
& \left\|T_{y_{1}}-T_{y_{2}}\right\|_{C\left[0, u_{0}\right]}=\sup _{u \leq u_{0}}\left|\int_{0}^{u} \varphi^{\left(y_{1}-y_{2}\right)}(z) e^{z m_{1}}(D(z))^{\left(m_{2}-1\right)} d z\right| e^{-u m_{1}}(D(u))^{-m_{2}} \\
& \quad \leq \text { const }\left\|y_{1}-y_{2}\right\|_{C\left[0, u_{0}\right]} \sup _{u \leq u_{0}}\left|\int_{0}^{u} z e^{z m_{1}}(D(z))^{\left(m_{2}-1\right)} d z\right| e^{-u m_{1}}(D(u))^{-m_{2}}
\end{aligned}
$$

and using the previous Lemma for $h(z):=z$

$$
\left(\int_{0}^{u} z e^{z m_{1}}(D(z))^{\left(m_{2}-1\right)} d z\right) e^{-u m_{1}}(D(u))^{-m_{2}}
$$

is of order $O(u)$ around zero. Thus $T$ is a contraction on $x$ for small enough $u_{0}$.

Step 2. Let us now prove that the continuous solution $y$ on $\left[0, u_{0}\right]$ is continuously differentiable on $\left[0, u_{0}\right)$. Notice that the function $\varphi^{(y)}$ is continuously differentiable when

- $\bar{F} \in C^{2}[0, \infty)$

- $P(u)$ is continuously differentiable on $\left[0, u_{0}\right)$

- $R^{\prime}(u)=\lambda \int_{0}^{\infty} \pi(x) f(x+u) d x<\infty$ on $\left[0, u_{0}\right)$ 
The following expansion for small $u$ is obtained

$$
\varphi^{(y)}(u)=a_{0}+a_{1} u+o(u), u \leq u_{0}
$$

where

$$
a_{0}=\Upsilon_{\alpha}(0)(\lambda \bar{F}(0)+\alpha)+R(0)+\alpha P(0)
$$

now using the latter in (16) along with the results of Lemma 4 yields

$$
\left(\int_{0}^{u} \varphi^{(y)}(z) e^{z m_{1}}(D(z))^{\left(m_{2}-1\right)} d z\right) e^{-u m_{1}}(D(u))^{-m_{2}}= \begin{cases}\frac{2 h(0)}{\sigma^{2}} u+o(u), & \sigma=0 \\ \frac{h(0)}{c}+o(u), & \sigma>0\end{cases}
$$

and $y$ is differentiable and it can also be proved that

$$
y^{\prime}(u)= \begin{cases}\frac{2}{\sigma^{2}} a_{0}+o(1), & \sigma=0 \\ \frac{2}{\sigma_{r}^{2}}\left(a_{1}-\frac{\delta a_{0}}{c}\right)+o(1), & \sigma>0\end{cases}
$$

for small valued $u$. And notice that for $\sigma>0$

$$
y(0)=\frac{(\lambda+\alpha) \pi\left(0^{-}\right)-\frac{\sigma^{2}}{2} \Upsilon_{\alpha}^{\prime \prime}(0)+R(0)+\alpha P(0)}{c}=0
$$

Step 3. Differentiating (16), a $C^{1}$-solution also solves (7).

Step 4. The extension of this local solution to $y \in C^{1}[0, \infty)$ is now introduced. Let us define $\xi(u):=y(u) D(u)$ and write (16) as a linear Volterra equation of the second kind

$$
\xi(u)=f_{1}(u)+\int_{0}^{u} f_{2}(u, z) \xi(z) d z
$$

where

$$
\begin{gathered}
f_{1}(u)=\Upsilon_{\alpha}(0) \int_{0}^{u}(\lambda \bar{F}(z)+\alpha) e^{-m_{1}(u-z)}\left(\frac{D(z)}{D(u)}\right)^{m_{2}-1} d z \\
+\int_{0}^{u}(R(z)+\alpha P(z)) e^{-m_{1}(u-z)}\left(\frac{D(z)}{D(u)}\right)^{m_{2}-1} d z \\
f_{2}(u, z)=(D(z))^{-1} \int_{z}^{u}(\lambda \bar{F}(x-z)+\alpha) e^{-m_{1}(u-x)}\left(\frac{D(x)}{D(u)}\right)^{m_{2}-1} d x
\end{gathered}
$$


$f_{1} \in C[0, \infty)$, and $f_{2}$ is also continuous, assuming that $P(u)$ and $R(u)$ are bounded on $[0, \infty)$. Thus

$$
\begin{aligned}
\xi(u) & =f_{1}(u)+\int_{0}^{u_{0}} f_{2}(u, z) \xi(z) d z+\int_{u_{0}}^{u} f_{2}(u, z) \xi(z) d z \\
& =g_{1}(u)+\int_{u_{0}}^{u} g_{2}(u, z) \xi(z) d z
\end{aligned}
$$

where $g_{1}$ and $g_{2}$ are continuous. The extension is proved using the same argument presented in Gaier \& Grandits (2005) on page 276.

Step 5. The solution $y$ is in $L^{1} \cap L^{\infty}$. The original function, using (6), can be given by

$$
\begin{aligned}
\Upsilon_{\alpha}(u) & =\mathbb{E}_{u}\left(P\left(U_{H_{\alpha}}\right) 1_{\left\{\tau \geq H_{\alpha}\right\}}+\pi\left(U_{\tau}\right) 1_{\left\{\tau<H_{\alpha}\right\}}\right) \\
& =\mathbb{E}_{u}\left(P\left(U_{H_{\alpha}}\right) 1_{\left\{\tau \geq H_{\alpha}\right\}}+\int_{U_{\tau^{-}}}^{\infty} \pi\left(U_{\tau^{-}}-z\right) f(z) d z 1_{\left\{\tau<H_{\alpha}\right\}}\right)
\end{aligned}
$$

where $U_{\tau^{-}}$is the so-called surplus previous to ruin. Assuming again $R(u)$ and $P(u)$ are bounded, $\Upsilon_{\alpha}(u)$ is bounded. Thus the integral $\Upsilon_{\alpha}(u)=\int_{0}^{u} y(z) d z$ is bounded and since $y \in C^{1}[0, \infty)$ the integrand function is also bounded.

The integrability at infinity issue is now addressed. It suffices to prove $\lim _{u \rightarrow \infty} y(u)=0$

$$
\begin{aligned}
\lim _{u \rightarrow \infty} y(u) & =\lim _{u \rightarrow \infty} \frac{\int_{0}^{u} \varphi^{(y)}(z) e^{z m_{1}}(D(z))^{\left(m_{2}-1\right)} d z}{e^{u m_{1}}(D(u))^{m_{2}}} \\
& =\lim _{u \rightarrow \infty} \frac{\Upsilon_{\alpha}(0)(\lambda \bar{F}(u)+\alpha)+\int_{0}^{u} y(u-x)(\lambda \bar{F}(x)+\alpha) d x+R(u)+\alpha P(u)}{m_{1} D(u)+m_{2}\left(\frac{\sigma_{r}^{2}}{2}\right)} \\
& =\lim _{u \rightarrow \infty} \frac{\Upsilon_{\alpha}(0)(\lambda \bar{F}(u)+\alpha)+\int_{0}^{u} y(u-x)(\lambda \bar{F}(x)+\alpha) d x+R(u)+\alpha P(u)}{m_{1} D(u)+m_{2}\left(\frac{\sigma_{r}^{2}}{2}\right)} \\
& =\lim _{u \rightarrow \infty} \frac{\Upsilon_{\alpha}(u)(\lambda+\alpha)-\lambda \int_{0}^{u} \Upsilon_{\alpha}(u) f(x) d x+R(u)+\alpha P(u)}{m_{1} D(u)+m_{2}\left(\frac{\sigma_{r}^{2}}{2}\right)}
\end{aligned}
$$

using integration by parts. Finally we may assume for $u$ large enough $\Upsilon_{\alpha}(u) \sim$ $P(u)$ and 


$$
\begin{gathered}
\lim _{u \rightarrow \infty} \frac{\Upsilon_{\alpha}(u)(\lambda+\alpha)-\lambda \int_{0}^{u} \Upsilon_{\alpha}(u-x) f(x) d x+R(u)+\alpha P(u)}{m_{1} D(u)+m_{2}\left(\frac{\sigma_{r}^{2}}{2}\right)} \\
=\lim _{u \rightarrow \infty} \frac{a R(u)+b P(u)}{u}=0
\end{gathered}
$$

for two scalars $a$ and $b$.

\section{ACKNOWLEDGEMENTS}

The authors gratefully acknowledge the financial support from Ministerio de Educación y Ciencia, Spain, Research Projects SEC2003-1828 and SEJ2004$01688 / \mathrm{ECON}$ and the useful comments from the referees.

\section{REFERENCES}

Asmussen, S. and Bladt, M. (1996) Phase-type distributions and Risk Processes with statedependent premiums. Scand. Act. Journal 1996, 19-36.

Abramowitz, M. and Stegun, I. (1970) Handbook of Mathematical Functions. 9th printing.

BERK, J. and GREen, R. (2004) Mutual Funds flows and performance in rational markets. Journal of Political Economy 112(6), 1269-1295.

CAI, J. and Dickson, D.C.M. (2002) On the expected discounted penalty function at ruin of a surplus process with interest. Insurance: Mathematics and Economics 30, 389-404.

CAI, J. (2004). Ruin probabilities and penalty functions with stochastic rates of interest. Stochastic Processes and Their Applications 112, 53-78.

CAI, J. and YANG, H.L. (2005) Ruin in the perturbed compound Poisson risk process under interest force. Advances in Applied Probability 37, 819-835.

Delbaen, F. and Haezendonck, J. (1987) Classical risk theory in an econmic environment. Insurance: Mathematics and Economics 6, 85-116.

DiCKSON, D.C.M. and WATERS, H.R. (1999) Ruin probability with compounding assets. Insurance: Mathematics and Economics 25, 49-62.

Duffie, D., Filipovic, D. and Schachermayer, W. (2003) Affine processes and aplications in finance. Annals of Applied Probability 13(3), 984-1053.

Embrechts, P. and Schmidli, H. (1994). Ruin estimations for a general insurance risk model. Advances in applied probability 26, 404-422.

Gaier, J. and Grandits, P. (2004) Ruin probabilities and investment under interest force in the presence of regularly varying tails. Scand. Actuarial Journal 4, 256-278.

GARRIDO, J. (1989) Stochastic diffrential equations for compound risk reserves. Insurance: Mathematics and Economics 8, 165-173.

Gerber, H. and Shiu, E. (1998) On the time value of ruin. North American Actuarial Journal 2, 48-78.

Gerber, H. and YANG, H. (2007) Absolute ruin probabilities ina jump diffusion risk model with investments. North American Actuarial Journal 11(3), 159-169.

Görng-JeAschke, A. and Yor, M. (2003) A survey and some generalizations of Bessel processes. Bernoulli 9(2), 313-349.

Gradshteyn, I.S. and Ryzhik, I.M. (1994) Table of Integrals, Series and Products. Fifth edition. Academic Press. 
Kalashnikov, V. and Norberg, R. (2002) Power tailed ruin probabilities in the presence of risky investments. Stochastic Processes and Their Applications 98, 211-228.

MA, J. and Sun, X. (2003) Ruin probabilities for insurance models involving investments. Scandinavian Actuarial Journal, 217-237.

NorberG, R. (1999) Ruin problems with assets and liabilities of diffusion type. Stochastic processes and their applications $\mathbf{8 1}, 255-269$.

Novikov, A. (2003) Martingales and First-Passage Times for Ornstein-Uhlenbeck Processes with a Jump Component. Theory of Probability and Its Applications 48(2), 288-303.

Paulsen, J. (1993) Risk theory in a stochastic environment. Stochastic processes and their applications 21, 327-361.

PAulsen, J. and GJessing, H.K. (1997a) Optimal choice of dividend barriers for a risk process with stochastic return on investments. Insurance: Mathematics and Economics 20, 215-223.

Paulsen, J. and GJessing, H.K. (1997b) Ruin theory with stochastic return on investments. Advances in Applied Probability 29, 965-985.

PAUlsen, J. (1998) Risk theory with compounding assets - a survey. Insurance: Mathematics and Economics 22, 3-16.

Paulsen, J., Kasozi, J. and Steigen, A. (2005) A numerical method to find the probability of ultimate ruin in the classical risk model with stochastic return on investments. Insurance: Mathematics and Economics 36, 399-420.

Peters, K. (1994) Exact and asymptotic solutions for the time-dependent problem in collective ruin I. SIAM J. Appl. Math. 54, 1761.

Segerdahl, C.O. (1942) Er einige risikotheoretische Fragestellungen. Skand. Aktuar Tidskr. 25, 43-83.

Sundt, B. and Teugels, J.L. (1995) Ruin estimates under interest force. Insurance: Mathematics and Economics 16, 7-22.

WANG, G. and WU, R. (2001) Distributions for the risk process with stochastic return on investments. Stochastic processes and their applications 95, 329-341.

Willmot, G. and Dickson, D. (2003) The Gerber-Shiu discounted penalty function in the stationary renewal risk model. Insurance: Mathematics and Economics 32, 403-411.

YUEN, K.C., WANG, G. and NG, W.K. (2004) Ruin probabilities for a risk process with stochastic return on investments. Stochastic processes and their applications 110, 259-274.

YUEN, K.C. and WANG, G. (2005) Some ruin problems for a risk process with stochastic interest. North American Actuarial Journal 9(3), 129-142.

\section{FLORIN AVRAM}

Department of Mathematics

Université de Pau

France

E-Mail: Florin.Avram@univ-pau.fr

Corresponding author:

Miguel UsáBel

Department of Business Administration

Universidad Carlos III de Madrid

Spain

E-Mail: usabel@emp.uc3m.es 